ISSN 2073-8994

www.mdpi.com/journal/symmetry

Article

\title{
Asymmetry and Symmetry in the Beauty of Human Faces
}

\section{Dahlia W. Zaidel * and Marjan Hessamian}

Department of Psychology, University of California, Los Angeles (UCLA), Los Angeles, CA, USA; E-Mail: marjan@ucla.edu

* Author to whom correspondence should be addressed; E-Mail: dahliaz@ucla.edu

Received: 22 January 2010; in revised form: 16 February 2010 / Accepted: 16 February 2010 / Published: 23 February 2010

\begin{abstract}
The emphasis in the published literature has mostly been on symmetry as the critical source for beauty judgment. In fact, both symmetry and asymmetry serve as highly aesthetic sources of beauty, whether the context is perceptual or conceptual. The human brain is characterized by symbolic cognition and this type of cognition facilitates a range of aesthetic reactions. For example, both art and natural scenery contain asymmetrical elements, which nevertheless render the whole effect beautiful. A further good case in point is, in fact, human faces. Normally, faces are structurally left-right symmetrical content-wise but not size-wise or function-wise. Attractiveness has often been discussed in terms of content-wise full-face symmetry. To test whether or not attractiveness can be gleaned only from the presence of left-right full-faces we tested half faces. Three separate groups of participants viewed and rated the attractiveness of 56 full-faces (women's and men's), their 56 vertical left hemi-faces and 56 vertical right hemi-faces. We found no statistically significant differences in the attractiveness ratings of full- and hemi-faces (whether left or right). Instead, we found a strong and significant positive correlation between the ratings of the hemi- and full-faces. These results are consistent with the view that the underpinning of human facial beauty is complex and that bilateral symmetry does not constitute a principle factor in beauty assessment. We discuss that the highly evolved human brain, compared to other animals, as well as symbolic and abstract cognition in humans enable a wide variety of aesthetic reactions.
\end{abstract}

Keywords: attractiveness; aesthetics; art; brain; mate selection; courtship displays; biology 


\section{Introduction}

Symmetry is both a conceptual and a perceptual notion associated with beauty-related judgments, even as it implies different things in a range of scholarly areas. In physics, symmetry is linked to beauty in that symmetry describes the invariants of nature, which, if discerned could reveal the fundamental, true physical reality (e.g., [1]). Indeed, the pursuit of uncovering symmetry-related structures is a dominant research strategy in modern physics [2,3]. In mathematics, the intellectual pursuit of the universal formulation of symmetry (Group Theory) has led to major discoveries in physics, and to Einstein's general relativity theory [4]. In chemistry, left-right balance is a critical component in the notion of symmetry; it refers to regular arrangements of molecules and the more symmetrical, the more aesthetic [5]. In biology, a dominant view is that left-right bilateral symmetry describes health and high genetic quality; deviations from symmetry in animals are assumed to spell disease, presence of parasites, poor fitness qualities and basis for rejection of a potential mate [6,7]. Whether or not animals have beauty-related responses upon seeing this symmetry is not known; beauty reactions themselves may be unique to humans. In art, symmetry refers to left-right, top-bottom balance (of forms, colors, lines, light, and so on) in the composition as a whole; it is an essential component of art's aesthetic quality [8]. In sum, symmetry is associated with beauty-related reactions and viewed as aesthetically pleasing in several scholarly domains.

However, absence of symmetry does not necessarily mean absence of beauty, and this is true in natural scenery, in art, or in human faces. In natural vistas such as sunsets over the Pacific Ocean in California, to use but one example, cloud formations consist of asymmetrical nonsense shapes, and yet the whole scene is highly aesthetically pleasing. In Japanese art, there is a long tradition espousing a type of aesthetic that idealizes asymmetry [9], a concept that is associated with Zen Buddhism in Japan [10]. The "... traditional Japanese aesthetics is an aesthetics of imperfection, insufficiency, incompleteness, asymmetry, and irregularity ..." [10] p. 88). This aesthetic where asymmetry in both art and nature is emphasized is also known as wabi-sabi [11] and Hacko [12]. A cracked ceramic jug, or an isolated dilapidated wooden hut, a cloudy sky with asymmetrical misshapen clouds, and asymmetrical flower arrangements are considered beautiful (see Figure 1). With human faces, attractiveness has been associated with left-right symmetry [13] as well as with asymmetry [14,15].

Similarly, in biology the evidence points in the direction of heritability of asymmetrical characteristics, which judging from the fossil record go back at least 350 million years [16]. Thus, the whole issue of symmetry in animals being a critical -component in biological reproductive strategies, mate selection, and survival of the species is doubtful [17]. Even in the case of the peacock's elaborate tail, the usefulness of symmetry for reproduction selection has recently been questioned [18,19], and equally doubted in other bird species [20]. Bees have been shown to successfully pollinate symmetrical as well as asymmetrical flowers [21]. Indeed, the published literature in biology is replete with examples of physical and functional asymmetries in animals [16,22]. Clearly then, factors other than symmetry also play a role in reproductive strategies. 
Figure 1. In a Japanese school of aesthetics asymmetry in art or nature is viewed as a strong source of beauty. The symmetrical figure in the bottom right panel surely does not invite higher aesthetic considerations than those in the remaining panels. The human brain with its symbolic and abstract cognition can reflect on the aesthetics of both symmetrical and asymmetrical patterns.

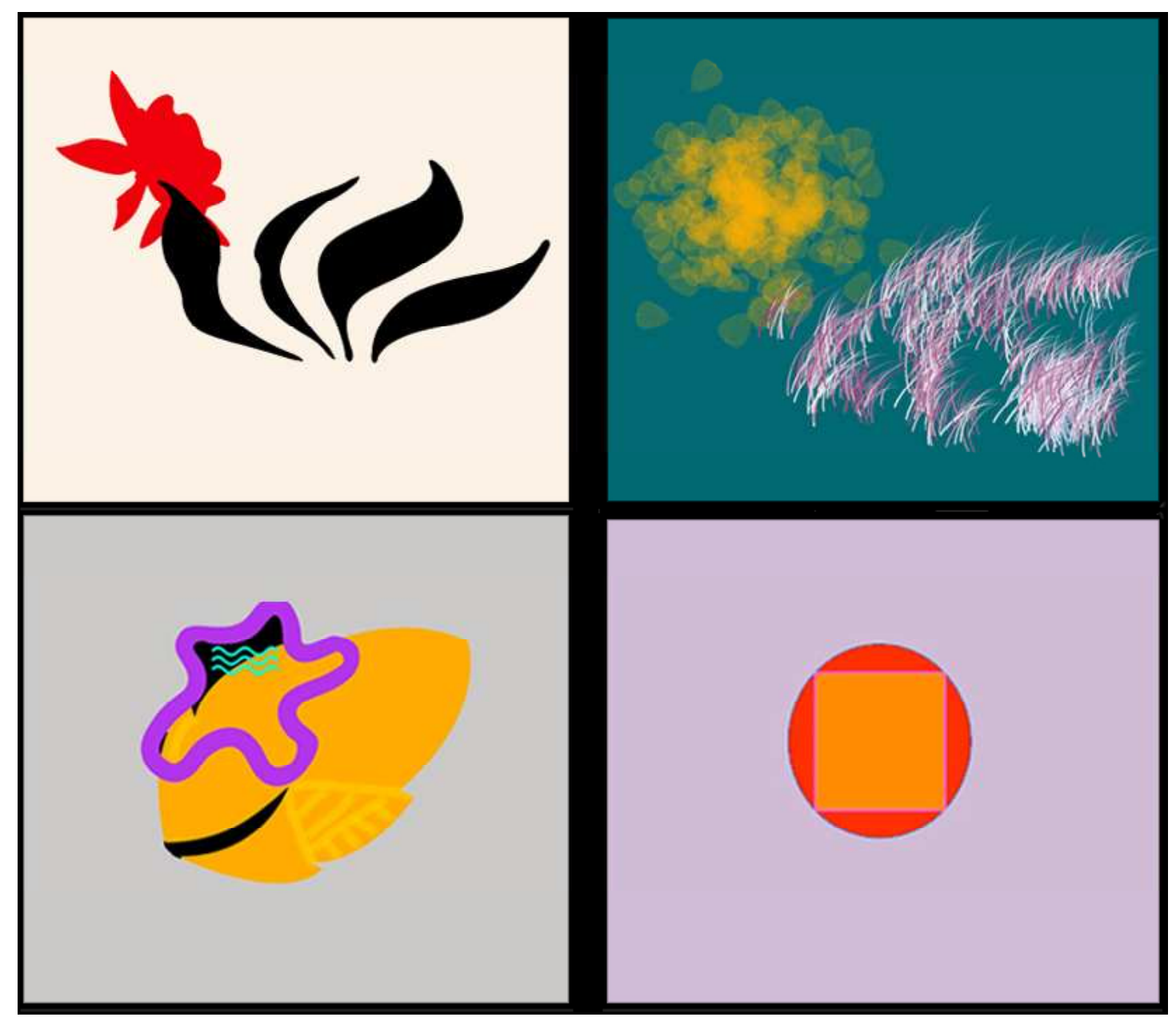

Fluctuating asymmetry and directional asymmetry are both important concepts in discussions of the phenomenon of symmetry in nature [16]. Both types can be inherited and have a genetic basis. Fluctuating asymmetry arises from random variations reflecting changes in the environment or in the response of the organism to the variation, such as toxins, climate change, food scarcity, and so on. These events serve as signals of physical fitness (because they are markers of survival abilities). In this view, with regards to attractiveness, the more symmetrical the face, the more attractive it is (that is, the greater the fitness). Compared to directional asymmetries, fluctuating asymmetries have mostly a 1:1 left-right ratio in the population and they are commonly relatively small [16]. Directional asymmetry refers to unequal structure size or function, as in handedness, hemispheric specialization, specific brain region size, organ placement within the body, or face side size, and the ratios are not 1:1. In fact, they are highly skewed. The human brain has evolved strong functional asymmetries (i.e., directional asymmetry) that are unique among animals, and they are not due to fluctuating asymmetries. Broadly speaking, the main speech and language regions are represented in the left hemisphere, and visuospatial cognition and face recognition are represented mostly in the right hemisphere [23,24]. 
Numerous other functional brain asymmetries have been reported [25]. A further central and critical difference between the brains of animals and humans is the prevalence of symbolic and abstract cognition in humans whereas it is nearly absent or minimal in animals [26,27]. The abstract symbolic thinking facilitates formation of infinite conceptual combinations (as in language, problem solving, insights, future plans, and art production), which explains partially why humans can entertain both symmetry and asymmetry as sources of beauty, regardless of whether or not the source is conceptual or perceptual [28].

The empirical focus of this paper is on the issue of symmetry and its relationship to attractiveness in human faces. Human faces are left-right asymmetrical with respect to size [29-33], micro-blood supply [34,35], and function [14,36-38]. Indeed, physical features in human faces have been found to be naturally and normally asymmetrical in numerous studies, whether using X-rays [39] or threedimensional morphometric measurements [33,40], or direct measurements [29]. Functional asymmetries have also been reported, particularly for smiling [14,41], speaking [42,43], and the appearance of beauty [14]. Perfectly bilaterally symmetrical faces created on a computer (not morphed, averaged, or digitally smoothed) were judged significantly less attractive than normal faces [15]. Even very beautiful faces belonging to fashion models were found to be somewhat asymmetrical [44].

Under normal circumstances, bilateral asymmetry in humans, rather than perfect left-right symmetry, was fashioned by millions of years of adaptive evolution and it implies perfection, not imperfection. One reasonable adaptive evolutionary scenario is that the face of the observer co-evolved with the brain of the beholder; as the human brain traded the perfect symmetry of its biological animal ancestors and slowly gained functional asymmetry over time, the face, too, gained physical and functional asymmetries to match the hemispheric functional specialization of the observer. Of course, other parallel evolutionary changes occurred over time as well, including social communication through close face-to-face proximity.

Fundamentally, mating and courtship signals are meant for the brain of the observer of the same species. To be effective, the signals need to be decoded by the brain of the observer. We need to assume that human-specific cognition enters into the neuronal calculations of human mate selection choices. Courtship displays by peacocks, birds of paradise, or butterflies are meaningless as far as attracting humans for reproduction, and the same applies to bowerbirds, or swallows, or rabbits, and so on. There is simplicity in ease of detection in the case of perceptual symmetry $[45,46]$, without even invoking the issue of aesthetics in the brain of the animal observer. While the perceptual simplicity allows for detection of deviations arising from, say, bodily parasite invasion, diseases, toxins, unsuccessful fights, and can be made by simple brains, the sophisticated, highly evolved asymmetric human brain, in contrast, can handle - and does - elaborate communication signals, including multiple symbols, metaphors, and cultural considerations. Moreover, the perceptual ease with which symmetry is detected has been shown empirically $[47,48]$ suggesting that genetic quality or fitness indicators that may be present in symmetry could not be the sole basis for mate selection. Rather, it is the perceptual simplicity interpreted by neuronally simple brain. Indeed, the notion that facial symmetry is a marker of health in humans has been questioned [49,50]. Although numerous studies with human faces have supported the link between attractiveness and symmetry (e.g., $[13,51,52])$, results in other studies have cast doubt on the link [14,15,44,53-56]. In humans, facial fitness indicators are yet to be fully understood against the background of general animal biological mate selection displays, in part 
because of the neuronal uniqueness of the human brain where the signals are interpreted, as well as the complexity of human societal, environmental, and cultural conditions (e.g., choice of a mate is determined by parents or match-makers) that do not exist in animal cultures, and in part because we do not yet have evidence that aesthetic judgment is present in animals.

We have shown previously that perfectly symmetrical computer constructed faces are rated less attractive than normal faces [15]. We wondered if viewing only half of a normal face would also be considered less attractive than the full face. A previous study by Scheib and colleagues [55] limited the question of hemi-face attractiveness to men's faces, restricted the ratings only to female participants, and focused on the issue of masculinity on the face. In the present study, we used full- and hemi-faces of both sexes as stimuli and asked both sexes to provide the attractiveness ratings. We were less interested in the appearance of masculinity or in actual physical dimensions of the full-faces (which was the interest in the Scheib et al. study [55]), than in determining participants' behavioral responses to the stimuli through attractiveness ratings. In daily life, we all frequently process incomplete visual views of faces (regardless of face sex), whether milling through a crowd, walking down a grocery aisle, scrutinizing people in a restaurant, or outdoors when blocked sunlight obscures half of the face. We have grown accustomed to inferring information from these partial views alone. Here, attractiveness ratings of straight head-on full-faces and their vertical hemi-faces were compared (see a diagrammatic illustration in Figure 2 and its legend). If facial symmetry spells beauty, then we would expect hemi-faces to obtain low attractiveness ratings and we would also expect a low correlation between the ratings of the full- and hemi-faces.

Figure 2. This is a hand-drawn picture illustrating a diagrammatic face. In the experiment, photographed faces (in grayscale) were used. The full-faces as well as the hemi-faces (left or right) were viewed separately, each by a different group of participants.

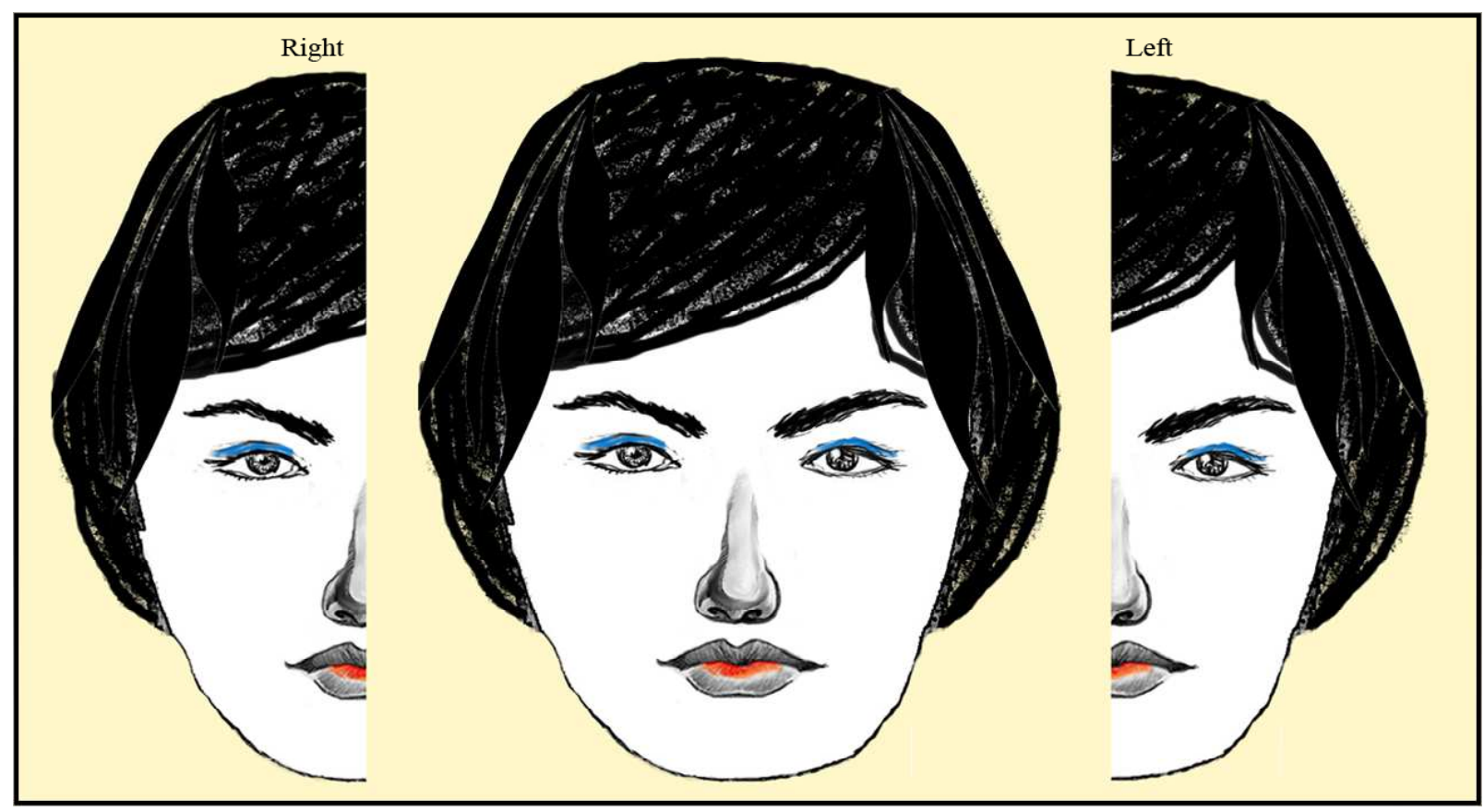




\section{Methods}

\subsection{Participants}

The participants were 82 (35 males, 47 females) undergraduate students from introductory psychology courses at the University of California, Los Angeles; they participated in the study in exchange for partial course credit. Of the 82 subjects, 27 (12 males, 15 females) participated in attractiveness rating of the full-faces, 29 different subjects (12 males, 17 females) rated the attractiveness of the left hemi-faces, and 26 different subjects (11 males, 15 females) rated the attractiveness of the right hemi-faces.

\subsection{Stimuli}

The faces were photographed in our lab or in a studio, under symmetrical lighting conditions, with uniform distance from the camera; subjects kept their gaze straight ahead at the camera, maintained front view (straight head-on), and had neutral expressions [see 14, 44, 49, 57]. The vertical left and right halves of each face were created with Photoshop software by dividing full-faces vertically down the facial midline center, after determining the mid-point of the inter-pupillary distance and the center of the philtrum in the upper lip (the infranasal groove). The grayscale stimuli consisted of the 56 front view full-faces (32 women, 24 men), their 56 right vertical hemi-faces detached from the full-faces as well as 56 left vertical hemi-faces (Figure 2 is a graphic illustration of a diagrammatic face and its two vertical hemi-faces).

\subsection{Procedures}

Participants were tested individually. The attractiveness rating of full-faces (56 stimuli), left-halves (56 stimuli), and right-halves (56 stimuli) was performed by three separate groups of participants (with no overlap in group participation). In each presentation set, the stimuli appeared one at a time on the computer screen (Macintosh) for exposure duration of 7 seconds each. Stimulus presentation order in a given stimulus set was randomized for each participant. Participants entered their ratings directly on the computer keyboard while the image (the stimulus) was exposed on the screen; they were instructed to rate the attractiveness of the stimulus on a 5-point Likert scale where "1" was not attractive at all and " 5 " was very attractive.

\section{Results}

The principle question concerned differences, if any, between hemi-faces and full-faces with regard to attractiveness. The mean attractiveness rating for each face in each of the three stimulus sets (fullfaces, left hemi-faces, right hemi-faces) was calculated. Each stimulus set was viewed by a different group of subjects. Absence of any substantial difference between the two halves or between the halves and the full-faces emerged: The mean attractiveness rating for full faces was 3.21 ( $\mathrm{SD}=0.97$, range $=$ 1.33-4.85), the mean attractiveness rating for left hemi-faces was 3.08 (SD $=1.02$, range $=1.23-4.63)$, and the mean for right hemi-faces was $3.08(\mathrm{SD}=0.99$, range $=1.58-4.46)$. 
Assessing the attractiveness ratings with an ANOVA with a between-subjects factor of Stimulus Face (full-face, left hemi-face, right hemi-face) revealed no main effects and no interactions, thus showing that the ratings for the three different stimulus sets were not statistically significant $(\mathrm{F}=0.610, \mathrm{p}<0.543)$.

The correlation between the attractiveness ratings of the hemi- and the full-faces was determined as well. A robust positive Pearson correlation between the attractiveness ratings of the full-faces and the left hemi-faces emerged $(r=0.933, p<0.0001)$ as well as between the full-faces and the right hemifaces $(r=0.937, p<0.0001)$. A graphic illustration of the relationship between the variables of hemifaces (on the $\mathrm{Y}$-axis) and the full-faces (on the $\mathrm{X}$-axis) is in the scatter plot with a best-fit line, in Figure 3.

Figure 3. A scatter plot of the attractiveness ratings. The Figure is an illustration of the high positive correlation between the attractiveness ratings for the 56 full-faces and their 56 left hemi-faces and 56 right hemi-faces.

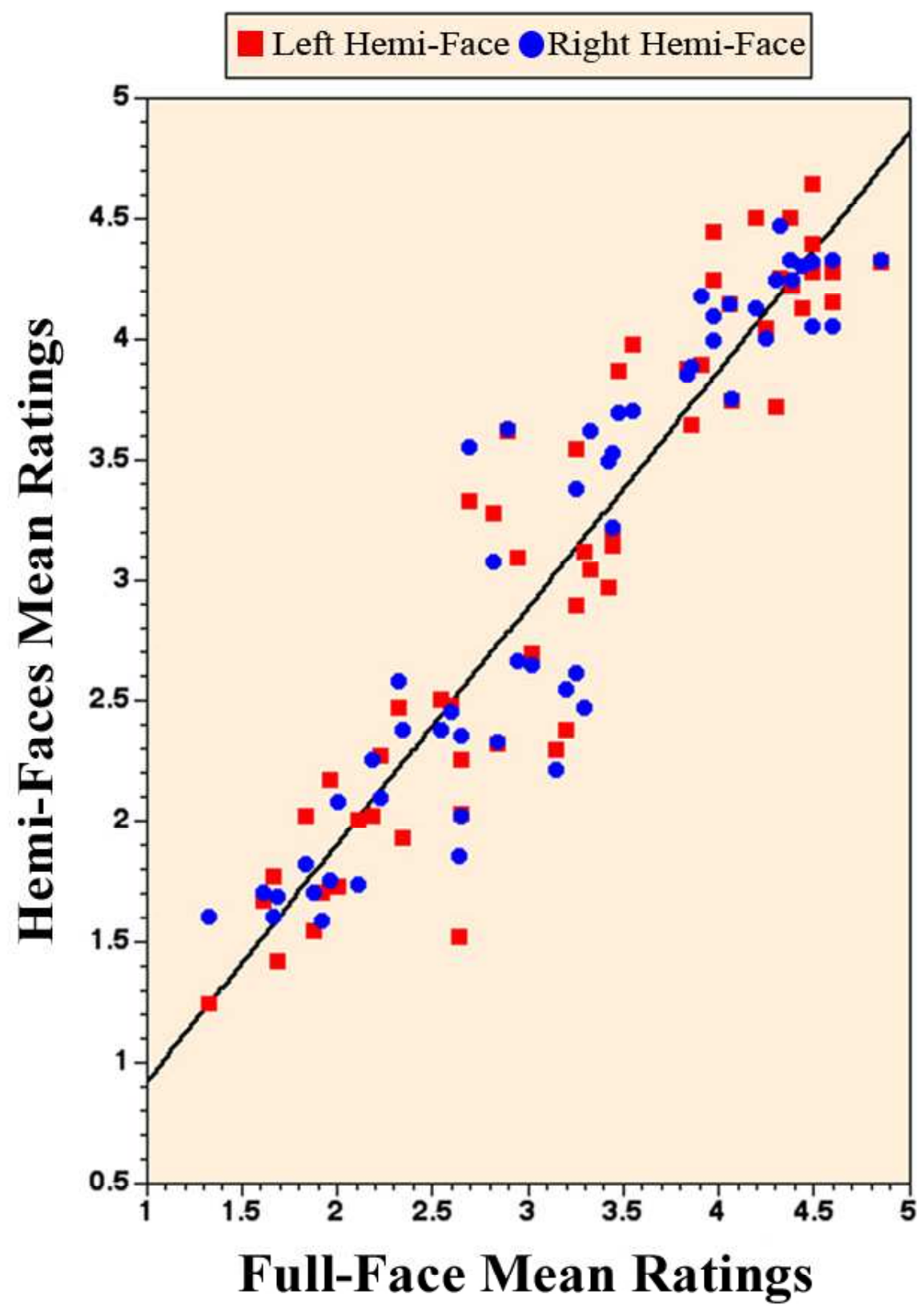




\section{Discussion}

Each stimulus set (full-faces, left hemi-faces, and right hemi-faces) was viewed by a different group of subjects. Hemi-faces of both women's and men's faces, regardless of whether they represented the left or the right halves, elicited attractiveness ratings not significantly different from full-faces. These data are consistent with results where only hemi-faces of men were rated and only female subjects participated [55], thus demonstrating that including both women and men, as done here, contributes to a balanced understanding of content-wise beauty appearance. One would have expected significantly higher ratings for the full-faces than the hemi-faces if bilateral symmetry were a principal component of attractiveness. The absence of such a difference in our study highlights the absence of left-right face symmetry as a critical factor in facial attractiveness. Indeed, previously, we have reported that perfectly symmetrical computer constructed full-faces were judged significantly less attractive than normal faces [15]. Moreover, in the present study, the correlation calculation revealed that the higher the attractiveness rating for the full-face, the higher the rating for its hemi-faces. Thus, components of beauty in both women's and men's faces are mostly wholly present in the vertical half of the face.

Assessing a hemi-face stimulus in a laboratory experiment is not unnatural [see also 55] considering that incomplete (obscured) views of faces are part of our normal daily visual experience, be it in a theater, restaurant, grocery store, classroom, or outdoors when shadows sometimes hide parts of the face. We have grown accustomed to inferring information about faces from these partial views alone. Whether or not we mentally "complete" the un-viewed part is a matter of debate and future empirical research. As emphasized above, each of the three stimulus sets was observed by a different group of subjects. Except for the group that viewed the full-faces, none of those who viewed hemi-faces had the benefit of "knowing" the attractiveness of the completed face (when asked informally, participants who viewed the hemi-faces commented they did not imagine the other half).

The notion that symmetry is a critical component in biological fitness advertisement originated with discussions of reproductive strategies in animals [7,58], and possibly was inspired by ideas of symmetry in physics and mathematics, since in those fields symmetry is considered as something akin to perfection. In theoretical physics, symmetry is viewed as the default universal design, and is often associated with mental concepts of beauty [1,3]. From the animal biological discussions there has been a generalization to human faces, although both symmetry (e.g., [13,52]) and asymmetry [14,15] have been linked with beauty, which suggests that perfect left-right symmetry is not critical in this context. To understand human reactions to facial attractiveness, we cannot ignore highly evolved humanspecific cognitions, their unique neuroanatomical underpinnings, and their role in human mate selection choice (see Introduction). Furthermore, whether or not animals experience beauty-related responses to the health-related symmetry they seek in their biological reproduction is not known.

The bulk of the studies advocating symmetry-equals-beauty in faces used digital morphing and averaging thereby erasing all natural physical asymmetries. While such studies have yielded important results, obviously they are not useful for zeroing in on the role of symmetry and facial beauty. Studies applying digital morphing and averaging commonly report that symmetry and attractiveness are positively correlated (e.g., [13,52]), while studies using techniques such as computer constructed leftleft (LL)/right-right (RR) faces find that bilateral symmetry is not relevant to attractiveness [14,15,44, $53,54,57,59,60]$. Neither approach is ideal since both deal with stationary faces in photographs. 
Morphing and averaging techniques also erase skin blotches, imperfections, moles, or discoloration, and thereby exaggerate facial attractiveness. The LL/RR approach allows natural structural asymmetries to remain in place.

Biological adaptive pressures sharpen the type, kind, and direction of reproductive fitness advertisements [16,61]. Sexually dimorphic traits in animals, such as long tails in peacocks (males) are examples of external traits developed in response to adaptive pressures associated with sexual and mate selection. Hormonal levels in both males and females also play a role in mate selection [62] and this extends to humans as well [63-65]. In all of these cases, displays are not random collections of signals. Rather, they are in large part expressions of the brain of both the displayer and of the observing potential mate [66,67], its evolutionary development over time, and many other different levels of adaptations. Even in the case of predators, there is a cost to the prey of having perfectly symmetrical coloration; the symmetry allows for easy detection by the predator [68]. With humans multiplicity of factors likely enter into selection of a mate for purposes of reproduction, with perfect facial symmetry not being a critical factor, since it would seem that an asymmetrical portion of the face, namely its hemi-face, displays beauty-related markers. In animals such as bees, where there is a high proportion of attraction to symmetrical flower shapes, there is also attraction to flowers with asymmetrical features without detrimental consequences to their reproductive biology [21]. Again, even here, we do not know if aesthetics is part of the biological formula for the attraction.

Furthermore, the presence of asymmetry in facial expressions did not originate with humans. Several studies of non-human primates have shown and discussed facial asymmetries in monkeys [69,70], marmosets [71], and chimpanzees [72], all of which can be viewed as precursors to human brain functional and anatomical asymmetries, as well as the beginnings of the co-evolution of human face and brain. Indeed, there is a myriad of behavioral asymmetries in animals (reviewed by Vallortigara and Rogers [73]), and those could not have developed without the brain of the perceiver being neuronally wired-up to perceive them.

Other aspects of faces that might influence mate selection in humans have been discussed [74-76]. In addition, several face studies addressing the issue of attraction and human mate selection have emphasized the importance of sex-related dimorphism and human facial symmetry $[14,77,78]$, averaged (digitally morphed) faces and symmetry [79], and facial masculinity and symmetry [55,63]. On balance, it would appear that both symmetry and asymmetry are features of the human face regardless of whether or not beauty is involved, and regardless of their relevance to mate selection.

The hallmark of human cognition compared to other animals, including non-human primates, is major reliance on symbolic, abstract, metaphorical thinking [27,28,80], and we cannot ignore that this type of cognition plays a substantial part in human mate choice. In normal face-on conversation, signals in the left half of the owner's face are processed initially in the observer's left hemisphere (via the owner's right visual field) and signals from the right half of the owner's face are initially processed in the observer's right hemisphere (via the left visual field) [14]; strong functional asymmetry and specialization in the left and right cerebral hemispheres characterize the human brain. While sexual selection, mate selection, and health-related reproduction in biology serve as guiding tools for research into human facial beauty, they alone cannot explain human facial attractiveness and the role it plays in human mate choice. This notion is supported by unique features in the human brain compared to other animals, as well as lack of evidence for aesthetic responses in animals. Indeed, the issue of aesthetic 
experience in animals remains an enigma. Hence, human-specific cognition (e.g., symbolic, metaphorical, abstract) and how it is related to aesthetic responses should be considered as a factor in mate selection choice. In all, when applied to normal human physical form, directional asymmetry (see in Introduction) in its various manifestation spells biological adaptive perfection.

\section{Conclusions}

While symmetry serves as a source of beauty in many scholarly domains, it is not an exclusive source of beauty-related responses in humans. Multiple sources give rise to aesthetic reactions, including asymmetrical features, be they in faces, art, nature scenery, or various intellectual domains. It is proposed that the fact that humans experience multiple beauty responses stems from major sophisticated symbolic and abstract cognition supported by the human brain. Indeed, aesthetics may be a uniquely human cognition; we do not yet have evidence that animals experience aesthetic responses, even when they selectively respond to symmetry in successful reproductive strategies. We need to consider that regardless of the origin and logic of symmetry in nature, the neuronal arrangements in the human brain support a wide range of positive and negative aesthetic responses independently of whether or not symmetry is perceptually or conceptually present, and independently of any relationship to mate selection strategies in the case of faces.

\section{Acknowledgments}

We thank anonymous reviewers for helpful comments on the manuscript.

\section{References and Notes}

1. Kosso, P., Symmetry arguments in physics. Stud. Hist. Philos. Sci. A 1999, 30, 479-492.

2. Engler, G. Aesthetics in science and art. Br. J. Aesthetics 1990, 30, $24-34$.

3. Zee, A. Fearful symmetry: The search for beauty in modern physics. Princeton University Press: Princeton, NJ, USA, 2007.

4. Engler, G. Einstein, his theories, and his aesthetic considerations. Int. Stud. Philos. Sci. 2005, 19, 21-30.

5. Muller, A. Chemistry: The beauty of symmetry. Science 2003, 300, 749-750.

6. Gould, J.L.; Gould, C.G. Sexual selection. Scientific American Library: New York, 1989.

7. Cronin, H. The ant and the peacock. Cambridge University Press: Cambridge, UK, 1992.

8. Jacobsen, T.; Schubotz, R.I.; Hofel, L.; Y., V.C.D. Brain correlates of aesthetic judgment of beauty. NeuroImage 2006, 29, 276-285.

9. Saito, Y. The Japanese aesthetics of imperfection and insufficiency. J. Aesthet. Art Critic. 1997, 55, 377-385.

10. Wicks, R. The idealization of contingency in traditional Japanese aesthetics. J. Aesthet. Edu. 2005, 39, 88-101.

11. Juniper, A., Wabi Sabi: The Japanese art of impermanence. Tuttle Publishing: Tokyo, Japan, 2003. 
12. Marra, M.F. A history of modern Japanese aesthetics. University of Hawaii Press: Honolulu, HI, USA, 2001.

13. Grammer, K.; Thornhill, R. Human (Homo sapiens) facial attractiveness and sexual selection: The role of symmetry and averageness. J. Com. Psychol. 1994, 108, 233-242.

14. Zaidel, D.W.; Chen, A.C.; German, C. She is not a beauty even when she smiles: possible evolutionary basis for a relationship between facial attractiveness and hemispheric specialization. Neuropsychologia 1995, 33, 649-655.

15. Zaidel, D.W.; Deblieck, C. Attractiveness of natural faces compared to computer constructed perfectly symmetrical faces. Int. J. Neurosci. 2007, 117, 423-431.

16. Babcock, L.E. Asymmetry in the fossil record. Euro. Rev. 2005, 13, 135-143.

17. Palmer, A.R. Waltzing with asymmetry. Biol. Sci. 1996, 46, 518-532.

18. Stevens, M.; Castor-Perry, S.A.; Price, J.R.F. The protective value of conspicuous signals is not impaired by shape, size, or position asymmetry. Behav. Ecol. 2008, 20, 96-102.

19. Takahashi, M.; Arita, H.; Hiraiwa-Hasegawa, M.; Hasegawa, T. Peahens do not prefer peacocks with more elaborate trains. Anim. Behav. 2008, 75, 1209-1219.

20. Dufour, K.W.; Weatherhead, P.J. Reproductive consequences of bilateral asymmetry for individual male red-winged blackbirds. Behav. Ecol. 1998, 9, 232-242.

21. Midgley, J.J.; Johnson, S.D. Some pollinators do not prefer symmetrically marked or shaped daisy (Asteraceae) flowers. Evol. Ecol. 1998, 12, 123-126.

22. Neville, A.C. Animal asymmetry. Edward Arnold: London,UK, 1976.

23. Sperry, R.W. Lateral specialization in the surgically separated hemispheres. In Neurosciences Third Study Program, Schmitt, F.; Worden, F., Eds. MIT Press: Cambridge, MA, USA, 1974; Vol. 3.

24. Zaidel, D.W. Different concepts and meaning systems in the left and right hemispheres. Psychol. Learn. Motiv. 2000, 40, 1-21.

25. Serrien, D.J.; Ivry, R.B.; Swinnen, S.P. Dynamics of hemispheric specialization and integration in the context of motor control. Nat. Rev. Neurosci. 2006, 7, 160-167.

26. Zilhao, J. The emergence of ornaments and art: An archaeological perspective on the origins of "behavioral modernity". J. Archaeol. Res. 2007, 15, 1-54.

27. Mace, R. On becoming modern. Science 2009, 324, 1280-1281.

28. Zaidel, D.W. Art and brain: insights from neuropsychology, biology and evolution. J. Anat. 2010, 216, 20-27.

29. Farkas, L.G.; Posnick, J.C.; Hreczko, T.M. Growth patterns of the face: a morphometric study. Cleft Palate Craniofac. J. 1992, 29, 308-315.

30. Ferrario, V. F.; Sforza, C.; Pogio, C. E.; Tartaglia, G. Distance from symmetry: a threedimensional evaluation of facial asymmetry. J. Oral Maxillofac. Surg. 1994, 52, 1126-1132.

31. Ferrario, V.F.; Sforza, C.; Miani, A., Jr.; Serrao, G. A three-dimensional evaluation of human facial asymmetry. J. Anat. 1995, 186 (Pt 1), 103-110.

32. Ferrario, V.F.; Sforza, C.; Ciusa, V.; Dellavia, C.; Tartaglia, G.M. The effect of sex and age on facial asymmetry in healthy subjects: a cross-sectional study from adolescence to mid-adulthood. J. Oral Maxillofac. Surg. 2001, 59, 382-388. 
33. Ferrario, V.F.; Sforza, C.; Dellavia, C.; Tartaglia, G.M.; Colombo, A.; Caru, A. A quantitative three-dimensional assessment of soft tissue facial asymmetry of cleft lip and palate adult patients. J. Craniofac. Surg. 2003, 14, 739-746.

34. Drummond, P.D.; Mirco, N. Staring at one side of the face increases blood flow on that side of the face. Psychophysiol. 2004, 41, 281-287.

35. Benedicic, M.; Dolenk, V.V.; Stefanovska, A.; Bosnjak, R. Left-right asymmetry of the facial microvascular control. Clin. Auton. Res. 2006, 16, 58-60.

36. Sackheim, H.A.; Weiman, A.L.; Forman, B.D. Asymmetry of the face at rest: Size, area and emotional expression. Cortex 1984, 20, 165-178.

37. Dopson, W.G.; Beckwith, B.E.; Tucker, D.M.; Bullard-Bates, P.C. Asymmetry of facial expression in spontaneous emotion. Cortex 1984, 20, 243-251.

38. Borod, J.C.; Haywood, C.S.; Koff, E. Neuropsychological aspects of facial asymmetry during emotional expression: a review of the normal adult literature. Neuropsych. Rev. 1997, 7, 41-60.

39. Skvarilova, B. Facial asymmetry: an X-ray study. Acta Chir. Plast. 1994, 36, 89-91.

40. Peck, S.; Peck, L.; Kataia, M. Skeletal asymmetry in esthetically pleasing faces. Angle Orthod. 1991, 61, 43-48.

41. Wylie, D.R.; Goodale, M.A. Left sided oral asymmetries in spontaneous but not posed smiles. Neuropsychologia 1988, 26, 823-832.

42. Jordan, T.R.; Thomas, S.M. Hemiface contributions to hemispheric dominance in visual speech perception. Neuropsychology 2007, 21, 721-731.

43. Nicholls, M.E.; Searle, D.A. Asymmetries for the visual expression and perception of speech. Brain Lang. 2006, 97, 322-331.

44. Zaidel, D.W.; Cohen, J.A. The face, beauty, and symmetry: perceiving asymmetry in beautiful faces. Int. J. Neurosci. 2005, 115, 1165-1173.

45. Wagemans, J. Characteristics and models of human symmetry detection. Trends Cogn. Sci. 1997, 1, 346-352.

46. Swaddle, J. P. Visual signaling by asymmetry: a review of perceptual processes. Proc. R. Soc. Lond., B, Biol. Sci. 1999, 354, 1383-1393.

47. Enquist, M.; Arak, A. Symmetry, beauty, and evolution. Nature 1994, 372, 169-172.

48. Johnstone, R.A. Female preference for symmetrical males as a by-product of selection for mate recognition. Nature 1994, 372, 172-175.

49. Reis, V.A.; Zaidel, D.W. Functional asymmetry in the human face: perception of health in the left and right sides of the face. Laterality 2001, 6, 225-231.

50. Zaidel, D.W.; Aarde, S.M.; Baig, K. Appearance of symmetry, beauty, and health in human faces. Brain Cogn. 2005, 57, 261-263.

51. Little, A.C.; Jones, B.C. Evidence against perceptual bias views for symmetry preferences in human faces. Proc. R. Soc. Lond., B, Biol. Sci. 2003, 270, 1759-1763.

52. Rhodes, G.; Proffitt, F.; Grady, J.M.; Sumich, A. Facial symmetry and the perception of beauty. Psychon. Bull. Rev. 1998, 5, 659-669.

53. Knowner, R. Facial asymmetry and attractiveness judgment in developmental perspective. J. Exp. Psychol. 1996, 22, 662-675. 
54. Samuels, C. A.; Butterworth, G.; Roberts, T.; Graupner, L.; Hole, G. Facial aesthetics: Babies prefer attractiveness to symmetry. Perception 1994, 23, 823-831.

55. Scheib, J.E.; Gangestad, S.W.; Thornhill, R. Facial attractiveness, symmetry and cues of good genes. Proc. R. Soc. Lond., B, Biol. Sci. 1999, 266, 1913-1917.

56. Springer, I.N.; Wannicke, B.; Warnke, P.H.; Zernial, O.; Wiltfang, J.; Russo, P.A.J.; Terheyden, H.; Reinhardt, A.; Wolfart, S. Visual attractiveness: Visual impact of symmetry increases significantly towards the midline. Ann. Plast. Surg. 2007, 59, 156-162.

57. Chen, A.C.; German, C.; Zaidel, D.W. Brain asymmetry and facial attractiveness: beauty is not simply in the eye of the beholder. Neuropsychologia 1997, 35, 471-476.

58. Moller, A.P. Bumblebee preference for symmetrical flowers. Proc. Nat. Acad. Sci. USA 1995, 92 , 2288-2292.

59. Langlois, J.H.; Roggman, L.A. Attractive faces are only average. Psychol. Sci. 1990, 1, 115-121.

60. Swaddle, J.P.; Cuthill, J.C. Asymmetry and human facial attractiveness: symmetry may not always be beautiful. Proc. R. Soc. Lond., B, Biol. Sci. 1995, 261, 111-116.

61. Ritchie, M.G. The shape of female mating preferences. Proc. Nat. Acad. Sci. USA 1996, 93, 14628-14631.

62. Ellison, P.T. Beauty: In the gonads of the beholder - and the beheld. Horm. Behav. 2008, 53, 1113.

63. Penton-Voak, I.S.; Little, A.C.; Jones, B.C.; Burt, D.M.; Tiddeman, B.P.; Perrett, D.I. Female condition influences preferences for sexual dimorphism in faces of male humans (Homo sapiens). J. Comp. Psychol. 2003, 117, 264-271.

64. Swaddle, J.P.; Reierson, G.W. Testosterone increases perceived dominance but not attractiveness in human males. Pro. Biol. Sci. 2002, 22, 2285-2289.

65. Welling, L.L.; Jones, B.C.; DeBruine, L.M.; Conway, C.A.; Law Smith, M.J.; Little, A.C.; Feinberg, D.R.; Sharp, M.A.; Al-Dujaili, E.A. Raised salivary testosterone in women is associated with increased attraction to masculine faces. Horm. Behav. 2007, 52, 156-161.

66. Madden, J. Sex, bowers and brains. Proc. R. Soc. Lond., B, Biol. Sci. 2001, 268, 833-838.

67. Keagy, J.; Savard, J.-F.; Borgia, G. Male satin bowerbird problem-solving ability predicts mating success. Anim. Behav. 2009, 100, 1-9.

68. Cuthill, I.C.; Hiby, E.; Lloyd, E. The predation costs of symmetrical cryptic coloration. Proc. $R$. Soc. Lond., B, Biol. Sci. 2006, 273, 1267-1271.

69. Hauser, M.D.; Akre, K. Asymmetries in the timing of facial and vocal expressions in rhesus monkeys: Implications for hemispheric specialization. Anim. Behav. 2001, 61, 391-408.

70. Weiss, D., Ghazanfar, A., Miller, C.T.; Hauser, M.D. Specialized processing of primate facial and vocal expressions: Evidence for cerebral asymmetries. In Cerebral vertebrate lateralization, Andrews, L.R.R., Ed. Cambridge University Press: New York, NY, USA, 2002.

71. Hook-Costigan, M.A.; Rogers, L.J. Lateralised use of the mouth in production of vocalizations by marmosets. Neuropsychologia 1998, 36, 1265-1273.

72. Fernandez-Cariba, S.; Loeches, A.; Morcillo, A.; Hopkins, W.D. Asymmetry in facial expression of emotions by chimpanzees. Neuropsychologia 2002, 40, 1523-1533.

73. Vallortigara, G.; Rogers, L.J. Survival with an asymmetrical brain: advantages and disadvantages of cerebral lateralization. Behav. Brain Sci. 2005, 28, 575-589. 
74. Buss, D.M. Strategies of human mating. Psychol. Topics 2006, 15, 239-260.

75. Rhodes, G. The evolutionary psychology of facial beauty. Annu. Rev. Psychol. 2006, 57, 199-226.

76. Yorzinski, J.L.; Platt, M.L. Same-sex gaze attraction influences mate-choice copying in humans. PLOS ONE 2010, 5, e9115.

77. Little, A.C.; Jones, B.C.; Waitt, C.; Tiddman, B.P.; Feinberg, D.R.; Perrett, D.I.; Apicella, C.L.; Marlowe, F.W. Symmetry is related to sexual dimorphism in faces: data across culture and species. Plos One 2008, 7, e2106.

78. Fink, B.; Neave, N.; Manning, J.T.; Grammer, K. Facial symmetry and judgements of attractiveness, health and personality. Pers. Individ. Dif. 2006, 41, 491-499.

79. Jones, B.C.; DeBruine, L.M.; Little, A.C. The role of symmetry in attraction to average faces. Percept. Psychophys. 2007, 69, 1273-1277.

80. Zaidel, D.W. Brain and art: Neuro-clues from intersection of disciplines. In Neuroaesthetics, Skov, M.; Vartanian, O., Eds. Baywood: Amityville, NY, USA, 2009.

(C) 2010 by the authors; licensee Molecular Diversity Preservation International, Basel, Switzerland. This article is an open-access article distributed under the terms and conditions of the Creative Commons Attribution license (http://creativecommons.org/licenses/by/3.0/). 